\title{
Bone disease in haemoglobin disorders
}

\author{
Ersi Voskaridou, ${ }^{1}$ Evangelos Terpos² \\ ${ }^{1}$ Thalassaemia Reference Center, Laikon General Hospital; ${ }^{2}$ Department of Clinical Therapeutics, \\ University of Athens School of Medicine, Athens, Greece
}

\begin{abstract}
Bone disease represents a prominent cause of morbidity in patients with thalassaemia and other haemoglobin disorders. The delay in sexual maturation, the presence of diabetes and hypothyroidism, the parathyroid gland dysfunction, the haemolytic anaemia, the progressive marrow expansion, the iron toxicity on osteoblasts, the iron chelators, and the deficiency of growth hormone or insulin growth factors have been identified as major causes of osteoporosis in thalassaemia. Adequate hormonal replacement, effective iron chelation, improvement of hemoglobin levels, calcium and vitamin D administration, physical activity, and smoking cessation are the main to-date measures for the management of the disease. During the last decade, novel pathogenetic data suggest that the reduced osteoblastic activity, which is believed to be the basic mechanism of bone loss in thalassemia, is accompanied by a comparable or even greater increase in bone resorption. Therefore, potent inhibitors of osteoclast activation, such as the aminobisphosphonates, arise as key drugs for the management of osteoporosis in thalassaemia patients and other haemoglobin disorders.
\end{abstract}

\section{Introduction}

\section{Thalassaemia and bone disease}

Thalassaemia major (TM) is a hereditary haemolytic anaemia caused by a defect in the ability of erythroblasts to synthesize the beta chain of adult haemoglobin. Bone disease in thalassaemia is manifested by diffuse bone pain, scoliosis, spinal deformities, nerve compression, spontaneous fractures and severe osteoporosis. As a rule, untreated TM patients present with severe bone deformities very early in life (1).

Correspondence: Ersi Voskaridou

(C) Copyright E. Voskaridou and E. Terpos, 2013

Licensee PAGEPress, Italy

Thalassemia Reports 2013; 3(s1):e20

doi:10.4081/thal.2013.s1.e20

This article is distributed under the terms of the Creative Commons Attribution Noncommercial License (by-nc 3.0) which permits any noncommercial use, distribution, and reproduction in any medium, provided the original author(s) and source are credited.

Parts of this work were presented at the

"3rd Pan-European Conference on Haemoglobinopathies and Rare Anaemias", Limassol (Cyprus), 24-26 October 2012.
In contrast, osteopenia and/or osteoporosis are major causes of morbitity in patients who survive longer as a result of improved treatment. The incidence of osteopenia or osteoporosis in well treated TM patients has been found to be approximately 40-50\%, and therefore osteoporosis represents a prominent cause of morbidity in TM patients of both genders (2).

The pathogenesis of osteoporosis in TM is very complicated and differs from the pathogenesis of bone deformities characteristically found in non transfused patients (thalassaemia intermedia; TI), who develop bone distortion mainly due to accelerated haemopoiesis and progressive marrow expansion. Several genetic and acquired factors are implicated in bone destruction in TM. The typical delay in sexual maturation, the presence of diabetes and hypothyroidism, the parathyroid gland dysfunction, the accelerated haemopoiesis with progressive marrow expansion, the direct iron toxicity on osteoblasts and the deficiency of growth hormone (GH) or insulin growth factor I (IGF-I) have been indicated as possible causes for thalassemia- induced osteoporosis (2-4). Furthermore, iron chelation has correlated with growth failure and bone abnormalities, and high desferrioxamine dosage has been associated with cartilage alterations $(5,6)$. More puzzling, however, is the observation that, despite the normalization of haemoglobin levels, adequate hormone replacement, and effective iron chelation, patients continue to show an unbalanced bone turnover with an increased resorptive phase resulting in seriously diminished bone mineral density (BMD) $(7,8)$.

Investigation of the mechanisms that underlie the bone pathology in TM has started rather late, in spite of the fact that the prominent facial and skull deformities, as well as the frequent fractures, were all noted in the original report of this disease by Cooley and Lee (1).

Bone is a complex organ with multiple functions. It provides the mechanical support for the body, it is a reservoir for normal mineral metabolism, and it is also the site of hematopoiesis (2). Bone is an active tissue constantly being remodeled and changing metabolically through the balanced activity of osteoclasts and osteoblasts on trabecular surfaces.

\section{Sickle cell disease and the bones}

Sickle cell disease (SCD) comprises a group of hereditary disorders characterized by sickling of erythrocytes when they are deoxygenated due to a single nucleotide change in the beta globin. Bone involvement is the commonest clinical manifestation of sickle cell disease.The bone in SCD is affected by microinfarction, osteopenia, osteoporosis, osteomyelitis, and osteonecrosis (9-11). The infarction of vertebral bone marrow and the presence of osteoporosis may produce collapse of the vertebrae with the typical fish mouth appearance. Several studies have shown an overall reduction in BMD in children and adults with SCD (9, 12-14). In recent studies, according to WHO criteria, 70-80\% of SCD patients were osteoporotic or osteopenic (15-17).

The causes of BMD abnormalities in patients with SCD are also multifactorial. Several factors, including reduced haemoglobin levels, chronic haemolytic anaemia, hyperplasia of the bone marrow, abnor- 
mally low body mass index (BMI), increased ferritin levels, vitamin D deficiencies and low plasma zinc or sex steroid levels, have been previously correlated with reduced BMD in adult patients with SCD (17-19). Furthermore, reduced physical activity and decreased circulating $\mathrm{GH}$ are likely contributing factors $(20,21)$.

However, the pathophysiology of bone disease in SCD is incompletely understood.

There are also a few studies of biochemical markers of bone turnover in relation to BMD in SCD patients. Bone formation markers (bonespecific alkaline phosphatase b-ALP; osteocalcin, OC) as well bone resorption markers (C-telopeptide of collagen type I, CTX) levels were found to be elevated $(16,17)$.

Osteopenia/osteoporosis may be one of the major public health problems in SCD patients, particularly if the onset takes place at an early age.

\section{Pathogenesis of osteoporosis in thalassemia}

According to the Word Health Organization, osteoporosis is a disease characterized by low bone mass and microarchitectural deterioration of bone tissue, leading to enhanced bone fragility and a consequential increase in fracture risk (15). The cut-off of 2.5 standard deviations below the normal mean in BMD for the respective age is used for the definition of osteoporosis, whereas the decrease of BMD between 1.5 and 2.5 standard deviations below the normal mean for the respective age is defined as osteopenia (22). The most important factors that are implicated in the pathogenesis of bone loss in thalassemia patients are described below.

\section{Genetic factors}

Genetic factors seem to play a role in the development of low bone mass and osteoporotic fractures. These factors have been implicated in the pathogenesis of postmenopausal osteoporosis, as regulator genes of BMD, but have not been studied thoroughly in thalassemia-induced osteoporosis. The polymorphism at the Spl site of the collagen type Ial (COLIA 1) gene (collagen type I is the major bone matrix protein) was studied by Wonke and colleagues (23), who found that approximately $30 \%$ of the TM patients were heterozygotes (Ss) and $4 \%$ were homozygotes (SS) for the Sp1 polymorphism. The authors have concluded that male patients with TM carrying the Sp1 mutation may develop severe osteoporosis of the spine and the hip more frequently than patients who do not carry this mutation. The COLIA 1 polymorphism has been associated with reduced BMD in postmenopausal osteoporosis, and predisposes women to osteoporotic fractures (24). The genes encoding collagen types Ia1 and Ia2 (COLIA 1 and COLIA 2, respectively) are also important candidates for the genetic regulation of BMD, as mutations that affect the coding regimens of these genes are implicated in the pathogenesis of osteogenesis imperfecta and osteoarthritis (25). The study of COLIA 1 polymorphism may help in identifying thalassemia patients who are at higher risk to develop osteoporosis and pathologic fractures (26).

Other genetic factors that have been reported to correlate with bone mineral damage in adult patients with -thalassemia include the vitamin D receptor (VDR) Bsml BB polymorphism, the loss- of-function mutations in the gene of the vitamin $\mathrm{D}$ receptor, the sequence variation of 713-8delC of transforming growth factor-1, the presence of restriction fragment length polymorphisms for the calcitonin (CT) receptor gene, estrogen receptor and interleukin-6 gene loci $(2,26)$. Although further studies are needed to exact final conclusion for the association between gene polymorphisms and bone mass in TM patients, COLIA 1 gene polymorphisms seem to be of importance in the pathogenesis of thalassemia- induced osteoporosis.

\section{Acquired factors}

Endocrine complications. Hypothyroidism, hypoparathyroidism, diabetes mellitus, and mainly hypogonadism (as delayed puberty and/or secondary hypogonadism) are considered as major causes of osteopenia/osteoporosis in TM $(2-4,6)$. Hemosiderosis of the pituitary gonadotrophic cells and iron deposition in the testes and ovaries are involved in the pathogenesis of endocrine complications in TM (27). Hypogonadism is a well-recognized cause of osteoporosis and osteopenia not only in patients with TM but also in the general population and is characterized by high bone turnover with enhanced resorptive phase (28). Estrogen and progesterone appear to inhibit osteoclast activity and pro- mote bone formation, whereas testosterone has a direct stimulatory effect on osteoblast proliferation and differentiation (4). IGFs play also an important role in bone remodeling. Low-serum IGF levels decrease osteoblast proliferation and bone matrix formation and reduce the activation of osteoclasts (29). Several studies have demonstrated a positive correlation between the BMD of the lumbar spine and the IGF-I concentration (30). It is well documented that the GH-IGF axis is defective in TM. Thalassemia patients have significantly lower circulating levels of IGF-I and the corresponding binding protein (IGFBP-III) than normal individuals; thus, leading to increased bone resorption, decreased bone formation, and finally to bone loss (31-32).

Iron overload and desferrioxamine. Iron deposition in the bone impairs osteoid maturation and inhibits mineralization locally, resulting in focal osteomalakia. The mechanism by which iron overload interferes in osteoid maturation and mineralization includes the incorporation of iron into crystals of calcium hydroxyapatite, which consequently affects the growth of hydroxyapatite crystals and reduces the bone metabolism unit tensile strength (33). Furthermore, desferrioxamine inhibits DNA synthesis, osteoblast, and fibroblast proliferation, osteoblast precursors differentiation, and collagen formation, although enhances osteoblast apoptosis, especially in patients who receive inappropriately high doses of desferrioxamine (34).

Bone marrow expansion. Bone marrow expansion, which is a typical finding in patients with TM, has been considered as a major cause of bone destruction (33). Transferrin receptor studies have demonstrated increased bone marrow activity even in patients with low reticulocyte count or marrow hypoplasia (35). However, there was found no direct correlation between serum levels of soluble transferrin receptor and the severity of osteoporosis (23).

Vitamin deficiencies. Vitamin C deficiency in iron- overload patients with low levels of serum ascorbic acid induces the risk of osteoporotic fractures (36). Vitamin D deficiency is also implicated in the pathogenesis of osteoporosis in TM patients due to the regulatory effect of vitamin D in both osteoclasts and osteoblasts. Adequate calcium intake and small amounts of vitamin D administration during skeleton development can increase bone mass in adolescents and decrease bone loss in adult life. However, most studies have failed to show reduced serum levels of 25-hydroxyvitamin D in TM patients.

Physical activity. Patients with TM have reduced physical activity due to the complications of the disease and the overprotection by their parents who do not encourage muscle activity. Thus, the lack of physical activity is another predisposing factor for osteoporosis in TM patients and muscle activity has to be encouraged in these patients $(2,4)$.

These factors can lead to the destruction of bone in thalassemia patients by increasing the osteoclast function and/or reducing the osteoblast activity.

Increased osteoclast function in thalassemic patients with osteoporosis. During the last decade, there was sufficient data supporting that increased osteoclast activation is present in TM patients. Patients with TM and osteoporosis have elevated markers of bone resorption, such as N-terminal cross-linking telopeptide of collagen type-I (NTX) and tartrate-resistant acid phosphatase type 5b (TRACP-5b) $(37,38)$ that correlated with BMD of the lumbar spine in these patients (38, 39 ). This increased osteoclast activity seems to be at least partially due to an imbalance in the receptor-activator of nuclear factor-kappa B ligand (RANKL)/osteoprotegerin (OPG) system and the overproduction of 
cytokines that are involved in the osteoclast differentiation and function (39). The RANK/RANKL/OPG pathway is of great importance for the activation and prolifera- tion of osteoclast precursors. We and others have shown that the ratio of sRANKL/OPG in the serum was increased in thalassemia patients with osteopenia/osteoporosis, providing evidence for the role of RANKL/OPG system in the pathogenesis of osteoporosis in thalassemia (39-41). Serum levels of IL-1, TNF- , IL6 , and TGF- , that are able to increase osteoclast function, were elevated in TM and correlated with bone resorption and lumbar BMD (42), suggesting their involvement in the pathogenesis of TM osteoporosis and supporting the role of the immune system in the bone loss of TM.

Reduced osteoblast function in thalassemic patients with osteoporosis. There is evidence of reduced osteoblast function in TM. Histomorphometry studies have revealed that increased osteoid thickness, increased osteoid maturation and min- eralization lag time, which indicate impaired bone matrix maturation, and defective mineralization is present in children and adolescents with TM (33). In addition, iron deposits appeared along mineralization fronts and osteoid surfaces, whereas focal thickened osteoid seams were found together with focal iron deposits $(33,43)$. Finally, dynamic bone formation histomorphometry studies established reduced bone formation rate in TM patients (33). This reduced bone formation is thought to-date to be mainly the result of iron poisoning in osteoblasts and/or the result of reduced function of $\mathrm{GH}$ and IGF-1 axis in TM patients. However, novel molecules seem to be implicated in osteoblast dysfunction in TM. Dickkopf-1 (Dkk-1) and sclerostin are Wnt signaling inhibitors that inhibit the osteoblast differentiation and function. We have recently shown that serum levels of Dkk-1 were increased in TM patients with osteoporosis and correlated with lumbar spine and wrist BMD. Interestingly, when zoledronic acid was given in these patients there was a reduction in Dkk-1 levels, which was not observed in the placebo group of this randomized trial (44). In another more recent study, we have shown that circulating sclerostin is elevated in thalassemia patients with osteoporosis and correlated with their BMD, but it was not reduced post zoledronic acid administration. The high circulating sclerostin and Dkk-1 levels and their association with BMD support the notion of a disrupted Wnt signaling in patients with thalassemia and osteoporosis which leads, in turn, to osteoblast deregulation. These findings give the rationale for the use of novel drugs targeting sclerostin and Dkk-1 in patients with thalassemia-induced osteoporosis.(45)

\section{References}

1. Cooley TB, Lee P. (1925) Series of cases of Splenomegaly in children with anaemia and peculiar bone changes. Trans Am Pediatr Soc. $37: 29$

2. Voskaridou E, Terpos E. (2004) New insights into the pathophysiology and management of osteoporosis in patients with beta thalassaemia. Br J Haematol. 127: 127-139.

3. Garofalo F, Piga A, Lala R, et al. (1998) Bone metabolism in thalassemia. Ann NY Acad Sci. 850: 475-478.

4. Voskaridou E, Terpos E. (2008) Pathogenesis and management of osteoporosis in thalassemia. Pediatr Endocrinol Rev. 6(Suppl. 1): 86-93.

5. Hatori M, Sparkman J, Teixeira CC, et al. (1995) Effects of deferoximine on chondrocyte alkaline phosphatase activity: proxidant role of deferoximine in thalassemia. Calcif Tissue Int. 57: 229-236.

6. Olivieri NF. (1999) The beta-thalassemias. N Engl J Med. 341: 99109.

7. Lasco A, Morabito N, Gaudio A, et al. (2001) Effects of hormonal replacement therapy on bone metabolism in young adults with beta-thalassemia major. Osteoporos Int. 12: 570- 575 .
8. Carmina E, Di Fede G, Napoli N, et al. (2004) Hypogonadism and hormone replacement therapy on bone mass of adult women with thalassemia major. Calcif Tissue Int. 74: 68-71.

9. Sarrai M, Duroseau H, D’Augustine J, et al. (2007) Bone mass density in adults with sickle cell disease. Br J Haematol. 136:666-672.

10. Voskaridou E, Stoupa E, Antoniadou L, et al. (2006) Osteoporosis and osteosclerosis in sickle cell/b-thalassemia: The role of the RANKL/osteoprotegerin axis. Haematologica. 91:813-816.

11. Miller RG, Segal JB, Ashar BH, et al. (2006) High prevalence and correlates of low bone mineral density in young adults with sickle cell disease. Am J Hematol. 81:236-241.

12. Gupta R, Marouf R, Adekile A. (2010) Pattern of bone mineral density in sickle cell disease patients with the high-HbF phenotype. Acta Haematol. 123: 64-70.

13. Brinker MR, Thomas KA, Meyers SJ, et al. (1998) Bone mineral density of the lumbar spine and proximal femur is decreased in children with sickle cell anemia. Am J Orthop., 27: 43-49.

14. Nelson DA, Rizvi S, Bhattacharyya T, et al. (2003) Trabecular and integral bone density in adults with sickle cell disease. J Clin Densitom. 6: 125-129.

15. World Health Organization. (1994) Assessment of fracture risks and its application to screening for post-menopausal osteoporosis. Report of a WHO Study Group. World Health Organ Tech Rep Ser. 843: 1-129.

16. Mohamed F, Elsha FM, Amna E, et al. (2012) The association of bone mineral density and parathyroid hormone with serum magnesium in adult patients with sickle-cell anaemia. Arch Med Sci. 2: 270-276.

17. Baldanzi G, Traina F, Marques Neto JF, et al. (2011) Low bone mass density is associated with hemolysis in brazilian patients with sickle cell disease. Clinics (Sao Paulo) 66: 801-805.

18. Claster S, Vichinsky EP. (2003) Managing sickle cell disease. $\mathrm{Br}$ Med J. 327: 1151-1155.

19. Al-Elq AH, Al-Turki HA, Sultan OA, et al. (2008). Influence of androgens on bone mass in young women with sickle cell anemia. Saudi Med. J. 29:980-983.

20. Adewoye AH, Chen TC, Ma Q, et al. (2008) Sickle cell bone disease: response to vitamin D and calcium. Am J Hematol. 83:271-274.

21. Fung BE, Kawchak AD, Zemel BS, et al. (2008) Markers of bone turnover are associated with growth and development in young subjects with sickle cell anemia. Pediatr Blood Cancer. 50: 620-623.

22. Binkley NC, Schmeer P, Wasnich RD, Lenchik L. (2002) What are the criteria by which a densitometric diagnosis of osteoporosis can be made in males and non-Caucasians? J Clin Densitom 5(Suppl.): 19-27.

23. Wonke B, Jensen C, Hanslip JJ, et al. (1998) Genetic and acquired predisposing factors and treatment of osteoporosis in thalassaemia major. J Pediatr Endocrinol MeTable 11(Suppl. 3): 795-801.

24. Uitterlinden AG, Weel AE, Burger $\mathrm{H}$, et al. (2001) Interaction between the vitamin $\mathrm{D}$ receptor gene and collagen type I alpha 1 gene in susceptibility for fracture. J Bone Miner Res. 16: 379-385.

25. Uitterlinden AG, Burger H, van Duijn CM, et al. (2000) Adjacent genes, for COL2A1 and the vitamin D receptor, are associated with separate features of radiographic osteoarthritis of the knee. Arthritis Rheum. 43: 1456-1464.

26. Perrotta S, Cappellini MD, Bertoldo F, et al. (2000) Osteoporosis in beta-thalassaemia major patients: analysis of the genetic background. Br J Haematol. 111: 461-466.

27. Berkovitch M, Bistritzer T, Milone SD, et al. (2000). Iron deposition in the anterior pituitary in homozygous beta- thalassemia: MRI evaluation and correlation with gonadal function. J Pediatr Endocrinol Metab 13: 179-184.

28. Riggs BL, Khosla S, Melton LJ 3rd. (1998) A unitary model for involutional osteoporosis: estrogen deficiency causes both type I and 
type II osteoporosis in postmenopausal women and contributes to bone loss in aging men. J Bone Miner Res 13: 763-773.

29. Geusens PP, Boonen S. (2002) 0steoporosis and the growth hormone-insulin-like growth factor axis. Horm Res. 58(Suppl. 3): 4955 .

30. Rucker D, Ezzat S, Diamandi A, et al. (2004) IGF-I and testosterone levels as predictors of bone mineral density in healthy, communitydwelling men. Clin Endocrinol. 60: 491-499.

31. Soliman AT, El Banna N, Abdel Fattah M, et al. (1998) Bone mineral density in prepubertal children with beta- thalassemia: correlation with growth and hormonal data. Metabolism. 47: 541-548.

32. Morabito N, Gaudio A, Lasco A, et al. (2004) Osteoprotegerin and RANKL in the pathogenesis of thalassemia- induced osteoporosis: new pieces of the puzzle. J Bone Miner Res. 19: 722-727.

33. Mahachoklertwattana P, Srikulchayanonta V, Chuansumrit A, et al. (2003) Bone histomorphometry in children and adolescents with beta-thalassemia disease: iron-associated focal osteomalacia. J Clin Endocrinol MeTable 88: 3966- 3972.

34. De Sanctis V, Pinamonti A, Di Palma A, et al. (1996) Growth and development in thalassaemia major patients with severe bone lesions due to desferrioxamine. Eur J Pediatr. 155: 368-372.

35. Ma ES, Lam KK, Chan AY, et al. (2003). Transferrin receptor-2 polymorphisms and iron overload in transfusion independent -thalassemia intermedia. Haematologica. 88: 345-346 [letter].

36. Michelson J, Cohen A. (1988) Incidence and treatment of fractures in thalassemia. J Orthop Trauma 2: 29-32.

37. Voskaridou E, Kyrtsonis MC, Terpos E, et al. (2001). Bone resorption is increased in young adults with thalassaemia major. Br $\mathrm{J}$ Haematol. 112: 36-41.
38. Voskaridou E, Terpos E, Spina G, et al. (2003). Pamidronate is an effective treatment for osteoporosis in patients with beta-thalassaemia. Br J Haematol. 123: 730-737.

39. Dresner Pollack R, Rachmilewitz E, Blumenfeld A, et al. (2000) Bone mineral metabolism in adults with beta- thalassaemia major and intermedia. Br J Haematol. 111: 902-907.

40. Voskaridou E, Terpos E. (2005) 0steoprotegerin to soluble receptor activator of nuclear factor kappa-B ligand ratio is reduced in patients with thalassaemia-related osteoporosis who receive vitamin D3. Eur J Haematol. 74: 359- 361.

41. Voskaridou E, Anagnostopoulos A, Konstantopoulos K, et al. (2006) Zoledronic acid for the treatment of osteoporosis in patients with beta-thalassemia: results from a single- center, randomized, placebo-controlled trial. Haematologica. 91: 1193-1202.

42. Morabito N, Russo GT, Gaudio A, et al. (2007) The "lively" cytokines network in beta-Thalassemia Major-related osteoporosis. Bone 40: 1588-1594.

43. Domrongkitchaiporn S, Sirikulchayanonta V, Angchaisuksiri P, et al. (2003) Abnormalities in bone mineral density and bone histology in thalassemia. J Bone Miner Res. 18: 1682-1688.

44. Voskaridou E, Christoulas D, Xirakia C, et al. (2009) Serum Dickkopf-1 is increased and correlates with reduced bone mineral density in patients with thalassemia-induced os- teoporosis. Reduction post-zoledronic acid administration. Haematologica. 94: 725-728.

45. Voskaridou E, Christoulas D, Plata E, et al. (2012) High circulating sclerostin is present in patients with thalassemia-associated osteoporosis and correlates with bone mineral density. Horm Metab Res. 44: $1-5$.

\section{Oral presentation}

Oral presentation is available online 